\title{
Topological considerations on microporous adsorption processes in simple models for pillared interlayered clays
}

\author{
N. G. Almarza, a) A. Gallardo, C. Martín, J. M. Guil, and E. Lomba \\ Instituto de Química-Física Rocasolano, CSIC, Serrano 119, E-28006 Madrid, Spain
}

(Received 23 September 2009; accepted 20 November 2009; published online 22 December 2009)

\begin{abstract}
The microporous structure of pillared interlayered clays is determined by their interlayer separation and the distribution of the pillars that separate their layers. The pillars provide stability to these quasi-two-dimensional high surface area materials. In this work we present a topological analysis of available and accessible volumes within various simple models of pillared interlayered clays. Each model is characterized by a distribution of pillars. Both fully ordered structures and disordered pillar distributions with either attractive or repulsive interpillar correlations are considered. Particular attention is paid to the problem of accessibility. In systems with similar degrees of porosity, even when cavities within each model might be able to host the same adsorbate molecules, their accessibility will strongly depend on the pillar distribution. The theoretical analysis presented in this work may facilitate the interpretation of experimental results, pointing out those quantities that are key to describe the texture of the porous material. (C) 2009 American Institute of Physics.
\end{abstract}

[doi:10.1063/1.3273209]

\section{INTRODUCTION}

Pillared interlayered clays (PILCs) belong to the class of disordered porous materials which exhibit both micro- and mesoporosities. These materials have been shown to have a great potential as adsorbents for gas separation ${ }^{1}$ and in catalysis. ${ }^{2}$ PILCs are obtained by transforming a mineral clay (usually unstable as regards its porous structure) into a chemically and thermally stable material by introducing pillars between the layers of the original layered structure. The process involves cation exchange between the pillaring agent and the layers, and a calcination process. ${ }^{3}$ The parent material is usually a cationic clay from the smectite group (layers are negatively charged) and the pillaring agent is typically a nano-oxide particle derived from a cationic polyoxohydroxy metal species (most frequently zirconium or aluminum oligomers).

In this type of materials, mesoporosity consists of pore voids between clay particles (whose sizes are of the order of a few microns). Microporosity, on the other hand, is determined by the spacing between clay layers, which can be tuned depending on the pillaring agent used and the parent material. ${ }^{4}$ Thus interlayer spacings ranging from 0.4 to $2 \mathrm{~nm}$ can easily be attained. On the other hand, the fine texture and adsorbing capabilities of the material strongly depend on the topological distribution and density of the pillars, which is mostly determined by the experimental conditions of the synthesis, ${ }^{1,5,6}$ and the layer charge of the parent clay. The density of pillars can be estimated from chemical analysis or from NMR spectroscopy. ${ }^{7,8}$ The analysis of the pillar distribution is a much more complex problem. In this connection, a variety of studies have focused on different alternatives to determine pore size distributions. In most cases one has to

${ }^{\text {a)} E l e c t r o n i c ~ m a i l: ~ n o e @ i q f r . c s i c . e s . ~}$ resort to fitting adsorption volume isotherms and/or microcalorimetric data to the corresponding isotherms of model materials composed of distributions of slit or cylindrical pores. $^{9-11}$ Additionally, the use of probe molecules of different sizes and shapes, comparing their adsorption isotherms in these systems with those in materials of well defined porous structure $^{12}$ (such as zeolites), can provide complementary information concerning the pore topology. Interpillar distances might be qualitatively estimated by means of this probe molecule approach. Use of small angle neutron scattering combined with contrast matching techniques has made possible to obtain more direct estimates of interpillar distances, ${ }^{13}$ but we are still far from solving the problem of providing even an statistical answer (e.g., in terms of pair distribution functions) to the question of how are the pillars distributed in these intercalated clays. It is reasonable to assume that the pillars will not be regularly distributed since during the intercalation process not all sites with cation exchange capacity will be substituted. Besides, the reaction conditions will also influence which sites are more suitable for pillar anchoring. In this context, the interactions between the pillar precursors prior to calcination will be another key factor as well.

In summary, PILCs will behave as quasi-twodimensional systems (e.g., modeling diffusion in PILCs requires the use of two-dimensional (2D) diffusion equations ${ }^{1}$ ) and the randomness in their pillar distributions can be thought of as a case of quenched disorder. This type of disorder poses considerable difficulties when interpreting experimental adsorption experiments. Therefore the aim of this paper is to provide a general topological analysis of various simple models of pillar configurations, ranging from fully ordered structures, to disordered systems with and without a certain degree of pillar aggregation. The analysis will focus on the comparison of available and accessible volumes for model adsorbates to different types of pillar configurations. 
For a given probe adsorbate molecule, available volume ${ }^{14}$ refers to the positions in the system that are not forbidden by steric restrictions due to the walls and/or the pillars. Particular attention will be paid to the estimation of accessible volume, defined as the fraction of available volume that can be accessed by a probe adsorbate molecule via internal diffusion. Our analysis will be based on the use of geometrical arguments and Monte Carlo (MC) approaches in conjunction with finite size scaling techniques in order to extrapolate our analysis to the thermodynamic limit.

The physics of adsorption in a medium characterized by quenched disorder translates into a nonequilibrium mixture problem in which one (or various) component(s) is(are) in thermal equilibrium and other component(s) has(have) one or several degrees of freedom frozen. This is nothing but a partly quenched system, which has posed a considerable challenge from the theoretical standpoint for many years. The pioneering work of Madden and Glandt ${ }^{15}$ and the formulation of the replica Orstein-Zernike (ROZ) theory by Given and Stell ${ }^{16}$ paved the way for a series of theoretical approaches focused on partly quenched systems. Particularly relevant to the class of models here considered is the extension of the ROZ equations to templated materials, ${ }^{17,18}$ and the inhomogeneous ROZ approach formulated by Pizio and Sokolowski, ${ }^{19}$ which accounts for the presence of topological disorder within slit pores. Porosity ${ }^{20}$ and connectivity analysis $^{21}$ have also been formulated in terms of integral equation approaches for disordered porous materials. Here, we will restrict ourselves so far to pursue a study based on geometrical considerations and $\mathrm{MC}$ analysis of the fine texture and adsorbing capabilities of a series of simple models of PILCs.

The rest of the paper is organized as follows. In Sec. II, the model is defined in terms of the interactions of the adsorbent with adsorbate molecules and the proposed model pillar configurations. In Sec. III the fraction of available volume is analyzed for various types of pillar configurations and its dependence on the adsorbate molecular size is assessed. Section IV is devoted to a detailed study of the accessible volume from different perspectives, analyzing both the connectivity of holes in terms of a Delaunay tessellation, and the percolation transition of barriers (pairs of pillars that block the diffusion of adsorbate molecules) that leads to pillar configurations that hinder the diffusion of a given adsorbate. Finally, in Sec. V, the analysis is applied to an experimental situation case study in which adsorption of probe molecules has been applied to qualitatively describe the porous structure of various PILCs. ${ }^{22}$

\section{THE MODEL}

The adsorbate molecules in our idealized PILC are confined by an external potential with the form

$$
V_{\text {ext }}\left(\mathbf{r}_{i}\right)=V_{w}\left(z_{i}\right)+V_{p}\left(x_{i}, y_{i} \mid\{\mathbf{R}\}_{p}\right),
$$

where $V_{w}$ (wall-molecule interaction) is given by

$$
V_{w}\left(z_{i}\right)=\left\{\begin{array}{cc}
0 ; & \sigma / 2<z_{i}<H-\sigma / 2 \\
\infty ; & z_{i} \notin[\sigma / 2, H-\sigma / 2],
\end{array}\right.
$$

where $H$ is the height of the galleries (the interlayer separation) and $\sigma$ is the adsorbate molecular diameter.

The contribution $V_{p}$ in Eq. (1) takes into account the excluded volume effect of the pillars. Also, in Eq. (1) $\{\mathbf{R}\}_{p}$ denotes the positions of the pillars, which herein are considered to be cylindrical and in principle are assumed to lack translational long range order on the $x y$-plane. The potential energy contribution $V_{p}$ at the position $\mathbf{r}_{i}$ is taken to be

$$
V_{p}\left(\mathbf{r}_{i} \mid\{\mathbf{R}\}_{p}\right)=\sum_{k=1}^{N_{p}} \mathcal{V}_{p}\left(\mathbf{r}_{i}, \mathbf{R}_{\mathbf{k}}\right),
$$

where $N_{p}$ is the number of pillars, $\mathbf{R}_{k} \equiv\left(X_{k}, Y_{k}\right)$ indicates the position of the $k$ th pillar, and

$$
\mathcal{V}_{p}\left(\mathbf{r}_{i}, \mathbf{R}_{k}\right)= \begin{cases}0 & \text { if } r_{i K} \geq\left(d_{p}+\sigma\right) / 2 \\ \infty & \text { if } r_{i K}<\left(d_{p}+\sigma\right) / 2\end{cases}
$$

where $d_{p}$ is the diameter of the pillar, and since in our model we only consider hard core repulsions, the distance $r_{i K}$ is calculated on the $x y$-plane projection, i.e.,

$$
r_{i K}=\left[\left(x_{i}-X_{k}\right)^{2}+\left(y_{i}-Y_{k}\right)^{2}\right]^{1 / 2} .
$$

We are interested in the determination of the fraction of volume available to adsorbate molecules for different pillar arrangements at a constant given pillar density. As mentioned in Sec. I, the disorder in these quasi-two-dimensional systems can be dealt with resorting to the so-called quenched randomness. ${ }^{23}$ This means that different sets of pillar configurations (realizations) will be considered to compute averaged properties. Each realization can be interpreted as the result of a quench of an equilibrium state of a system of $2 \mathrm{D}$ particles, which are nothing but the $x y$-projection of the pillars. In practice, this means that explicit pillar configurations will be extracted from a Metropolis-MC ${ }^{23,24}$ simulation in the canonical ensemble of a hard core model in two dimensions at certain values of the model parameters and the corresponding pillar density. This quenching process can be thought of as a very rough idealization of the calcination step in the synthesis of PILCs, by which the pillars are definitely anchored to the layers and the final structure of the PILC is stabilized. $^{3}$

Our equilibrium model to extract pillar configurations is defined by a configurational energy

$$
U_{q}=\sum_{i=1}^{N_{p}-1} \sum_{j=i+1}^{N_{p}} \mathcal{V}_{p p}\left(R_{i j} \mid \sigma_{p}, K, \kappa, R_{c}\right),
$$

where the subindex $q$ stresses the fact that the purpose of this model is just to serve as a device to build up well characterized disordered pillar structures corresponding to particular realizations (quenches) of a given equilibrium distribution. For the pair interaction term $\mathcal{V}_{p p}$, we have chosen a hard core Yukawa potential, which is a function of the distance between disks (pillars), depending on the specific parameters, $K, \kappa, \sigma_{p}$, and $R_{c}$ as follows: 
$\mathcal{V}_{p p}(R)= \begin{cases}\infty & \text { if } R<\sigma_{p} \\ V_{Y}\left(R \mid \sigma_{p}, K, \kappa\right)-V_{Y}\left(R_{c} \mid \sigma_{p}, K, \kappa\right) & \text { if } \sigma_{p} \leq R \leq R_{c} \\ 0 & \text { if } R>R_{c},\end{cases}$

$\mathcal{V}_{Y}\left(R \mid \sigma_{p}, K, \kappa\right)=-K \frac{\sigma_{p}}{R} \exp \left[-\kappa\left(\frac{R}{\sigma_{p}}-1\right)\right]$,

with $\kappa>0$. Within a given selection of parameters and pillar density, the probability of a given configuration for the pillars will be defined by

$$
\Pi\left(\{\mathbf{R}\}_{p}\right) \propto \exp \left[-U_{q}\left(\{\mathbf{R}\}_{p} \mid \sigma_{p}, K, \kappa, R_{c}\right)\right] .
$$

In these equations $|K|$ plays the role of the inverse temperature, $\sigma_{p}$ is the minimum allowed distance between pillars (in most cases can be assumed to be equal to the diameter of the cylinder, $d_{p}$ ), $R_{c}$ is the pair potential cutoff distance (which is introduced for numerical convenience), and $\kappa$ is a parameter that controls the decay of the interactions. If $K=0$ the interaction potential reduces to that of hard disks. When $K>0$ each particle attracts neighboring particles, and this situation locally increases density fluctuations with respect to the hard disk case. In the disordered porous media this implies that larger cavities will appear, while at the same time some regions might become inaccessible to adsorbate molecules due to the accumulation of pillars.

In fact the value of $K$ cannot be increased arbitrarily, since phase separation (in principle a liquid-vapor equilibrium) can occur for $K>K_{c}$ ( $K_{c}$ being the critical inverse temperature, which depends on $\kappa$ and $\left.R_{c}\right){ }^{25}$ In these conditions, the porous structure (in particular, its size distribution and the distribution of pillar positions) derived from quenched configurations will show a considerable systemsize dependence.

On the other hand, for $K<0$ the distance between neighboring pillars tends to be larger than that of the hard disk case, and the configurations thus generated approach to some extent the topology of an ordered lattice of pillars. Two particular configurations for $K>0$ and $K<0$ are shown in Fig. 1. One immediately observes that larger cavities are present when the pillar configuration corresponds to a quench of attractive particles.

It is obvious that these choices to define the pillar distribution are somewhat arbitrary. But given the lack of detailed information as to the pillar distribution functions, and the complexity of the processes and interactions at play during the synthesis of real PILCs, we have resorted to probe these simple alternatives that take into account at least excluded volume effects and the possibility of varying degrees of pillar aggregation.

\section{AVAILABLE VOLUME FOR ADSORBATE MOLECULES}

Let us consider a gallery of dimensions $L \times L \times H$. The available volume for adsorbate molecules of diameter $\sigma$ in the limit of small pillar density will be given by

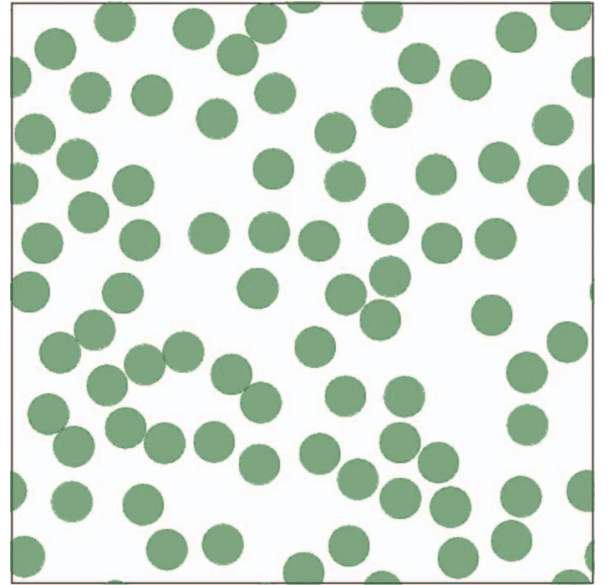

(a)

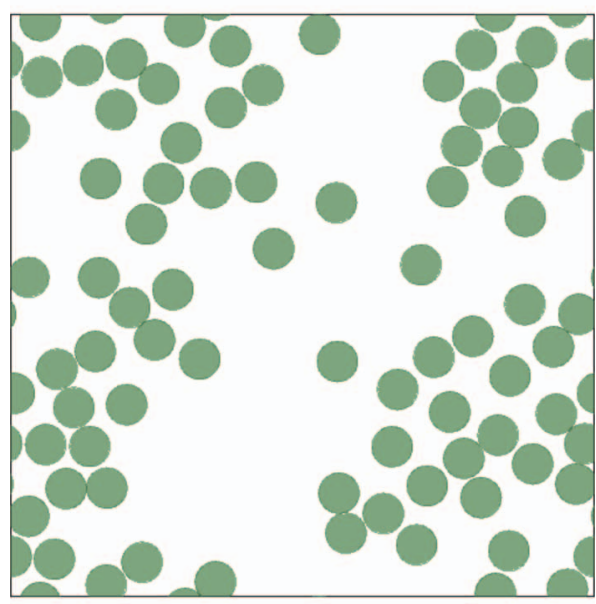

(b)

FIG. 1. Disordered configuration of pillars obtained by a quench of an equilibrium distribution of hard Yukawa disks with (a) repulsive and (b) attractive interactions. Labels correspond to the Yukawa coupling constant [see Eq. (8)].

$$
V_{\mathrm{av}}=(H-\sigma)\left[L^{2}-N_{p} \pi\left(d_{p}+\sigma\right)^{2} / 4\right]
$$

where $L$ is the length of the system in $x$ and $y$ directions. We define the parameter $\Phi_{\mathrm{av}}$ (fraction of available volume in the presence of pillars) as

$$
\Phi_{\mathrm{av}}=\frac{V_{\mathrm{av}}}{L^{2}(H-\sigma)} .
$$

In the limit of low density of pillars $\rho_{p} d_{p}^{2} \ll 1$ (with $\rho_{p}$ $\left.\equiv N_{p} / L^{2}\right)$, we get

$$
\Phi_{\mathrm{av}} \approx 1-\frac{\pi \rho_{p} d_{p}^{2}}{4}\left(1+\frac{\sigma}{d_{p}}\right)^{2} ; \quad \rho_{d} d_{p}^{2} \rightarrow 0 .
$$

When the density of pillars is not too low, and $\sigma / d_{p}>0$ (i.e., our adsorbate molecules have a finite size), the amount of available volume will depend on the particular pillar configuration. In principle, each pillar excludes the presence of adsorbates in an area $a_{1}$ around its location with 
TABLE I. Available volume fraction $\phi_{\mathrm{av}}$ for reduced pillar density $\rho d_{p}^{2}$ $=0.50$ and different pillar configurations.

\begin{tabular}{ccccc}
\hline \hline$\sigma / d_{p}$ & Square lattice & $K=1(\mathrm{AD})$ & $K=0(\mathrm{HS})$ & $k=-1(\mathrm{RD})$ \\
\hline 0.000 & $1-\pi / 8 \approx 0.6073$ & $1-\pi / 8$ & $1-\pi / 8$ & $1-\pi / 8$ \\
0.100 & 0.5248 & 0.527 & 0.526 & 0.527 \\
0.200 & 0.4345 & 0.449 & 0.446 & 0.444 \\
0.300 & 0.3363 & 0.376 & 0.370 & 0.365 \\
0.400 & 0.2303 & 0.311 & 0.300 & 0.292 \\
0.500 & 0.145 & 0.254 & 0.237 & 0.227 \\
\hline \hline
\end{tabular}

$$
a_{1}=\frac{\pi}{4}\left(d_{p}+\sigma\right)^{2} .
$$

Nevertheless, as $\rho_{p}$ increases, the exclusion areas due to different pillars can overlap. The available volume fraction can then be written as

$$
\begin{aligned}
\Phi_{\mathrm{av}}= & 1-\frac{1}{L^{2}} N_{p} a_{1}+\frac{1}{L^{2}} \sum_{k=1}^{N_{p}-1} \sum_{l=k+1}^{N_{p}} S_{2}\left(\mathbf{R}_{k}, \mathbf{R}_{l}\right) \\
& -\frac{1}{L^{2}} \sum_{k} \sum_{l>k} \sum_{m>l} S_{3}\left(\mathbf{R}_{k}, \mathbf{R}_{l}, \mathbf{R}_{m}\right)+\cdots,
\end{aligned}
$$

where $S_{2}\left(\mathbf{R}_{k}, \mathbf{R}_{l}\right)$ is the overlap between the exclusion areas due to pillars $k$ and $l$, and $S_{3}$ corresponds to triple overlaps. Equation (14) can be expressed in terms of pillar-pillar correlations and the interaction between the adsorbate and the pillars. $^{20,26}$ Taking into account that the distance between pairs of pillars must fulfill $R_{k l}>\sigma_{p}$ (we will take $\sigma_{p}=d_{p}$ ), the series in Eq. (14) can be truncated for small values of $\sigma / d_{p}$. In any case, it will be possible to compute estimates of $\Phi_{\mathrm{av}}$ resorting to $\mathrm{MC}$ integration strategies. ${ }^{24,27}$

Summarizing, if $\sigma / d_{p}>0$, the amount of available volume for the adsorbate depends not only on $\rho_{p}$, but also on the pillar distribution. As an example, we will consider a system with reduced pillar density $\rho d_{p}^{2}=0.50$ (with $\sigma_{p}=d_{p}$ ), and we will compute $\Phi_{\mathrm{av}}$ for adsorbates of different sizes. Several pillar distributions will be considered: (1) an ordered square lattice; (2) a hard disk distribution; (3a) hard disk Yukawalike pillar distributions with $\kappa=1, R_{c}=3 \sigma_{p}$, and $K=1$ [attractive disorder (AD)]; and (3b) the same with $\kappa=1, R_{c}=3$, and $K=-1$ [repulsive disorder (RD)]. In Table I the results for the available volume fraction for several adsorbate sizes are shown.

The results in Table I indicate that the disorder in the pillar structure increases the available volume, and this effect is further enhanced in the presence of $\mathrm{AD}$ (in which pillars are likely to group). The results shown in Table I were computed by MC integration (insertion tests) over representative configurations of each system. The simulations of the Yukawa models were carried out using $N_{p}=200$ and a volume (area) $A=400 \sigma_{p}^{2}$.

\section{ACCESSIBLE VOLUME FOR ADSORBATE MOLECULES}

In Sec. III we have analyzed the available volume of the adsorbent for a given adsorbate molecule. In the calculation we have only taken into account whether inside the adsor-

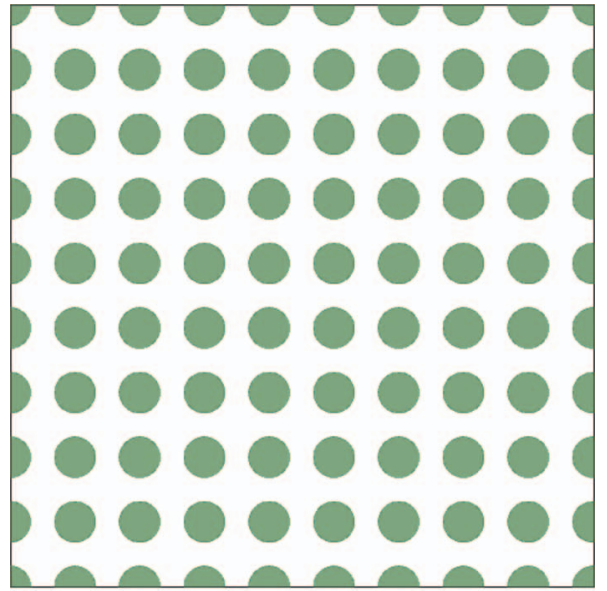

(a)

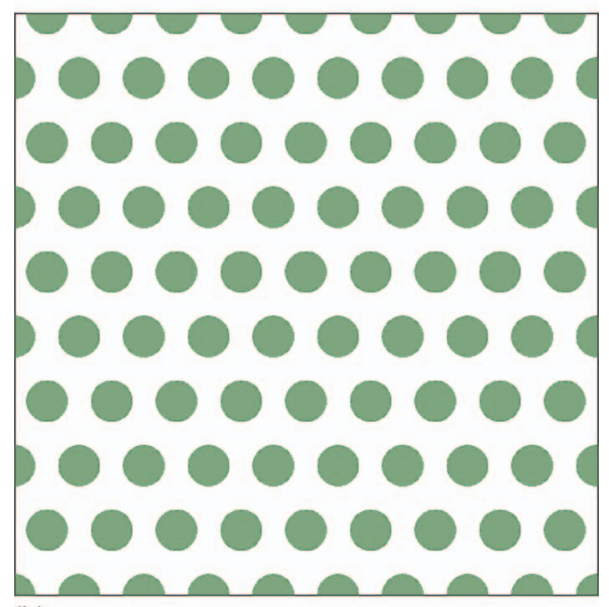

(b)

FIG. 2. Ordered configuration of pillars according to square and triangular bidimensional lattices.

bent there are cavities large enough to host a given adsorbate molecule. In an experimental situation adsorbate molecules need also a path to reach those cavities from the adsorbent external surface. This is the problem that we will address in this section, starting from the simplest situation in which the pillars follow a regular distribution on the sites of triangular and square lattices (see Fig. 2).

\section{A. An illustration with ordered porous structures 1. Pillars in a triangular lattice}

In this lattice each pillar occupies the node of a triangular lattice and is therefore surrounded by six nearest neighbors, located at a distance given by

$$
R_{n n} / d_{p}=\left[\frac{2}{\sqrt{3} \rho_{p} d_{p}^{2}}\right]^{1 / 2} .
$$

The maximum diameter $\sigma_{a}$ of a hard molecule that can enter or diffuse into the porous medium will therefore be limited by the width of the gate defined by two neighboring pillars,

$$
\sigma_{a}=R_{n n}-d_{p},
$$




$$
\frac{\sigma_{a}}{d_{p}}=\left[\frac{2}{\sqrt{3} \rho_{p} d_{p}^{2}}\right]^{1 / 2}-1 .
$$

A cavity in this regular system can be defined as the space between three nearest neighbor pillars (forming an equilateral triangle). Assuming that $H$ is much larger than $R_{n n}$, the maximum size of a spherical hard molecule $\sigma_{f}$ that fits in the cavity will be

$$
\sigma_{f}+d_{p}=\frac{2 \sqrt{3}}{3} R_{n n} .
$$

Using the same reduced density as in the previous calculations, $\rho_{p} d_{p}^{2}=0.50$, we get

$$
\frac{\sigma_{f}}{d_{p}}=\frac{2^{3 / 2}}{3^{3 / 4}\left(\rho_{p} d_{p}^{2}\right)^{1 / 2}}-1
$$

Using Eqs. (17) and (19) we get for $\rho_{p} d_{p}^{2}=0.50$,

$$
\frac{\sigma_{f}}{d_{p}} \simeq 0.755 \quad \frac{\sigma_{a}}{d_{p}} \simeq 0.520 .
$$

\section{Pillars in a square lattice}

In this case each pillar has four nearest neighbors at a distance

$$
R_{n n} / d_{p}=\left[\frac{1}{\rho_{p} d_{p}^{2}}\right]^{1 / 2} .
$$

A cavity for this lattice will be the space inside a square of four pillars. In this case we will have, for $H \gg R_{n n}$,

$$
\sigma_{f}+d_{p}=\sqrt{2} R_{n n},
$$

therefore, we get

$$
\begin{aligned}
& \sigma_{a} / d_{p}=\left[\frac{1}{\rho_{p} d_{p}^{2}}\right]^{1 / 2}-1, \\
& \sigma_{f} / d_{p}=\left[\frac{2}{\rho_{p} d_{p}^{2}}\right]^{1 / 2}-1,
\end{aligned}
$$

and explicitly for $\rho_{p} d_{p}^{2}=0.50$,

$$
\frac{\sigma_{f}}{d_{p}} \simeq 1.000 \quad \frac{\sigma_{a}}{d_{p}} \simeq 0.4142 .
$$

These previous two examples illustrate that the effects of the accessibility of the adsorbates must be taken into account when the pillar density is not low. In fact, the presence of larger cavities on a given adsorbent does not guarantee that larger molecules can be adsorbed, since the access of adsorbate molecules may be hindered by smaller gate widths.

\section{B. Accessible volume in disordered systems}

In Sec. IV A we have seen how the size of adsorbates entering the adsorbent is limited by the distance between neighboring pillars in the ordered lattices. The same situation is expected to happen when dealing with disordered porous media. Let us consider a given realization of pillar positions in our system: cavities of different sizes can be found. Now, we focus on an adsorbate probe molecule of diameter $\sigma$ located inside the porous media (i.e., it does not overlap with either the external walls or the pillars). According to our simple model for PILCs, the diffusion of this molecule along the $z$-direction is hindered by the flat walls. In $x$ - and $y$-directions, the diffusion is, at least in principle, possible. However, if the pillar density is high enough, it might happen that the diffusion of molecules of a given size along macroscopic distances inside the porous material becomes impossible.

Let us consider two locations inside the pore. In none of them the adsorbate molecule of size $\sigma$ overlaps with walls or pillars. The question arising is then, can our adsorbate molecule diffuse from one location to the other?

Let $\mathbf{r}_{1}$ and $\mathbf{r}_{2}$ denote the projections on the $x y$-plane of the two positions referred above. In principle, we could find a number of (possible) trajectories joining $\mathbf{r}_{1}$ and $\mathbf{r}_{2}$. In this 2D space we can consider that (for a given molecular size, $\sigma$ ) positions $\mathbf{r}_{1}$ and $\mathbf{r}_{2}$ belong to the same cavity ${ }^{28}$ if there exists a path $\mathcal{P}$ that connects both positions and fulfills for every point in the path

$$
V_{p}\left(x, y \mid\left\{\mathbf{R}_{p}\right\}\right)=0 \quad \forall(x, y) \in \mathcal{P} .
$$

The condition given in Eq. (26) will not be satisfied for trajectories passing close to any pillar position. In this case, by "close to" we mean that there is at least one point $(x, y)$ in the trajectory $\mathcal{P}$ that fulfills $\sqrt{\left(x-X_{k}\right)^{2}+\left(y-Y_{k}\right)^{2}} \leq\left(d_{p}+\sigma\right) / 2$, where $\left(X_{k}, Y_{k}\right)$ designates the $x y$-plane coordinates of the pillar under consideration. Depending on the size of the adsorbate molecule, it may happen that there is no possible trajectory linking $\mathbf{r}_{1}$ and $\mathbf{r}_{2}$ that fulfills Eq. (26).

Consider now two pillars placed at $\mathbf{R}_{k}$ and $\mathbf{R}_{l}$ with $R_{k l}$ $<d_{p}+\sigma$. Let us denote as $L_{k l}$ the segment joining these two pillars. It is clear that the possible trajectories of adsorbates with diameter $\sigma$ cannot cross $L_{k l}$, since any point belonging to $L_{k l}$ lies at a distance shorter than $\left(d_{p}+\sigma\right) / 2$ to at least one of the pillars that define the segment. We will denote these segments defined by pairs of pillars that fulfill $L_{k l}<d_{p}+\sigma$ as barriers.

When a certain number of barriers are linked and enclose a given region of the porous medium, this region defines an isolated cavity. If we have to analyze the results of an adsorption experiment, we should bear in mind that the volume-in principle available - belonging to regions completely fenced in by barriers cannot be reached by the adsorbates. A simple minded particle insertion test would not take into account the inaccessibility of this volume. Moreover, for a given pillar distribution we can expect that there will be a certain percolation threshold adsorbate molecular diameter that delimits the maximum size of the molecules that can fully penetrate the porous media. In what follows, we will address this issue.

\section{Percolation transition}

Let us consider that we have a model PILC with pillar configurations defined according to a certain probability distribution, and we want to compute the sizes of the adsorbates 
TABLE II. Adsorbate percolation diameters for pillar structures defined in terms of (i) a hard sphere fluid $(K=0)$, (ii) an attractive hard core Yukawa system $(K=1)$, and (iii) a repulsive hard core Yukawa interaction $(K=-1)$ at reduced density $\rho_{p} d_{p}^{2}=0.50$ for different system sizes.

\begin{tabular}{ccccccc}
\hline \hline$K$ & $L / \sigma_{p}$ & $\bar{\sigma}_{\mathrm{per}}^{(1)} / d_{p}$ & $\bar{\sigma}_{\mathrm{per}}^{(2)} / d_{p}$ & $\bar{\sigma}_{\mathrm{per}} / d_{p}$ & $\delta \sigma_{\mathrm{per}}^{(1)}$ & $\delta \sigma_{\mathrm{per}}^{(2)}$ \\
\hline 0 & 10 & $0.3634(2)$ & $0.4422(2)$ & $0.4028(2)$ & $0.0583(1)$ & $0.0705(1)$ \\
& 20 & $0.3759(4)$ & $0.4227(6)$ & $0.3993(4)$ & $0.0383(3)$ & $0.0430(3)$ \\
& 30 & $0.3808(4)$ & $0.4155(4)$ & $0.3981(3)$ & $0.0294(3)$ & $0.0321(3)$ \\
& 40 & $0.3844(6)$ & $0.4119(9)$ & $0.3981(7)$ & $0.0241(4)$ & $0.0259(5)$ \\
& 50 & $0.3858(5)$ & $0.4089(5)$ & $0.3973(7)$ & $0.0207(3)$ & $0.0220(5)$ \\
& 60 & $0.3868(5)$ & $0.4079(6)$ & $0.3973(4)$ & $0.0184(3)$ & $0.0194(4)$ \\
1 & & & & & \\
& 10 & $0.3397(3)$ & $0.4438(3)$ & $0.3917(3)$ & $0.0691(1)$ & $0.0948(3)$ \\
& 20 & $0.3526(7)$ & $0.4154(7)$ & $0.3840(5)$ & $0.0474(3)$ & $0.0584(5)$ \\
& 30 & $0.3585(11)$ & $0.4050(12)$ & $0.3817(8)$ & $0.0371(3)$ & $0.0432(10)$ \\
& 40 & $0.3617(11)$ & $0.3989(13)$ & $0.3803(10)$ & $0.0308(4)$ & $0.0351(7)$ \\
& 50 & $0.3636(19)$ & $0.3964(27)$ & $0.3800(22)$ & $0.0270(7)$ & $0.0305(10)$ \\
& 60 & $0.3646(14)$ & $0.3942(17)$ & $0.3794(13)$ & $0.0245(12)$ & $0.0273(14)$ \\
& & & & & \\
& 10 & $0.3758(1)$ & $0.4428(2)$ & $0.4093(1)$ & $0.0519(1)$ & $0.0596(1)$ \\
& 20 & $0.3874(2)$ & $0.4268(2)$ & $0.4071(2)$ & $0.0334(2)$ & $0.0363(2)$ \\
& 30 & $0.3921(4)$ & $0.4211(5)$ & $0.4066(4)$ & $0.0253(2)$ & $0.0271(2)$ \\
& 40 & $0.3943(5)$ & $0.4175(6)$ & $0.4059(5)$ & $0.0208(2)$ & $0.0219(3)$ \\
& 50 & $0.3958(5)$ & $0.4157(9)$ & $0.4057(5)$ & $0.0179(3)$ & $0.0187(5)$ \\
& 60 & $0.3973(7)$ & $0.4146(5)$ & $0.4059(3)$ & $0.0156(3)$ & $0.0162(3)$ \\
\hline \hline
\end{tabular}

that can penetrate the porous media. We will apply periodic boundary conditions in order to remove boundary effects.

The analysis of a given configuration can be sketched as follows.

- Distances between all pillar pairs (or at least those which are relatively close) are computed and stored. Then, ordered lists (according to the interpillar distance) containing pairs of pillars and distances between them are constructed.

- Clusters are created establishing links between pillar pairs that are separated by a distance $R \leq R_{\mathrm{cl}}$ (geometric criterion), and the structure of the clusters is analyzed in terms of the reference distance $R_{\mathrm{cl}}$.

- We determine the first (smallest) reference distance $R_{\mathrm{cl}}=R_{\mathrm{per}}^{(1)}$, at which a cluster percolates through the periodic system. Adsorbates with $\sigma>R_{\mathrm{per}}^{(1)}-d_{p}$ will not be able to diffuse over the whole porous system (they could still diffuse in one direction). This means that we have defined a cluster of barriers that percolates throughout the sample.

- An additional reference distance can be determined, $R_{\mathrm{cl}}=R_{\text {per }}^{(2)}$, at which barrier percolation occurs in a different direction to the one defined by $R_{\text {per }}^{(1)}$. Adsorbates with $\sigma>R_{\text {per }}^{(2)}-d_{p}$ will then be trapped in closed regions of the porous media.

Now, for a given model of disorder characterized by a set of parameters $\left\{K, \kappa, R_{c}, \sigma_{p}=d_{p}\right\}$, and a certain pillar density, $\rho_{p} d_{p}^{2}$, we perform simulations for different system sizes $L$, this being the side length of the square simulation box. From the simulation results we can compute the averages of the adsorbate percolation diameters: $\bar{\sigma}_{\mathrm{per}}^{(1)} \equiv\left\langle R_{\mathrm{per}}^{(1)}\right\rangle-d_{p}, \bar{\sigma}_{\mathrm{per}}^{(2)}$ $\equiv\left\langle R_{\mathrm{per}}^{(2)}\right\rangle-d_{p}$, and their corresponding fluctuations over the sampling of different pillar configurations: $\delta \sigma_{\text {per }}^{(i)}=\left[\left\langle\left(\sigma_{\text {per }}^{(i)}\right)^{2}\right\rangle\right.$ $\left.-\left\langle\sigma_{\text {per }}^{(i)}\right\rangle^{2}\right]^{1 / 2}$. These quantities are expected to depend on the system size $L$. At the thermodynamic limit $(L \rightarrow \infty)$, one should have $\delta \sigma_{\text {per }}^{(i)} \rightarrow 0$ and $\left(\bar{\sigma}_{\text {per }}^{(2)}-\bar{\sigma}_{\text {per }}^{(1)}\right) \rightarrow 0$.

Let us recall at this point that our goal is to analyze the effect of the pillar disorder on the ability of the adsorbent to host adsorbates of different sizes. To this purpose we have carried out simulations and performed the barrier percolation analysis for the three classes of disorder-hard disks ( $K$ $=0), \mathrm{AD}(K=1)$, and $\mathrm{RD}(K=-1)$-and various system sizes. The most relevant results of the percolation analysis are collected in Table II.

As $L$ increases, all systems behave as expected: the fluctuation of the percolation diameter shrinks and $\bar{\sigma}_{\text {per }}^{(1)} \rightarrow \bar{\sigma}_{\text {per }}^{(2)}$. Consequently, at least in these particular cases, it seems that $\sigma_{\text {per }}=\left(\sigma_{\text {per }}^{(1)}+\sigma_{\text {per }}^{(2)}\right) / 2$ might be used as an appropriate observable to estimate the percolation threshold. It is worth noticing that these disorder models produce lower limiting sizes for the accessible adsorbates than the ordered structures considered in Sec. IV A.

Now, according to percolation theory, ${ }^{29}$ the effective percolation threshold for finite systems of size $L$ follows:

$$
\sigma_{\text {per }}(L)-\sigma_{\text {per }}(\infty) \propto L^{-1 / \nu},
$$

where $\nu$ is a critical exponent. The value of the percolation exponent $\nu$ for $2 \mathrm{D}$ systems is ${ }^{14,29} \nu=4 / 3$. In order to take into account corrections to scaling, we have fitted the numerical results to equations of the form 
TABLE III. Thermodynamic limit extrapolation of the adsorbate percolation diameters for pillar density $\rho_{p} d_{p}^{2}=0.50$ (raw data in Table II). The error estimates are given as twice the standard deviation of the mean in units of the last figure quoted.

\begin{tabular}{llll}
\hline \hline Model & \multicolumn{1}{c}{$\sigma_{\mathrm{per}}^{(1)} / d_{p}$} & \multicolumn{1}{c}{$\sigma_{\mathrm{per}}^{(2)} / d_{p}$} & \multicolumn{1}{c}{$\sigma_{\mathrm{per}} / d_{p}$} \\
\hline$K=0$ & $0.3963(13)$ & $0.3964(16)$ & $0.3963(11)$ \\
$K=1$ & $0.376(3)$ & $0.378(4)$ & $0.377(3)$ \\
$K=-1$ & $0.4045(4)$ & $0.4054(11)$ & $0.4051(8)$ \\
\hline \hline
\end{tabular}

$$
\sigma_{\mathrm{per}}^{(i)}(L)=\sigma_{\mathrm{per}}^{(i)}+\sum_{k=1}^{n_{k}} a_{k}\left(L^{-3 / 4}\right)^{k}
$$

The results shown in Table II are well represented by Eq. (28) with $n_{k}=2$. The estimates of $\sigma_{\mathrm{per}}^{(i)}$ and $\sigma_{\text {per }}$ from these fittings can be found in Table III. Taking into account the extrapolated values for $\sigma_{\mathrm{per}}^{(i)}$ and $\sigma_{\mathrm{per}}$ for the different types of configurations, we get as final percolation threshold estimates (for the pillar density $\rho_{p} d_{p}^{2}=0.50$ )

$$
\begin{aligned}
& \sigma_{\mathrm{acc}}(K=0) / d_{p}=0.396 \pm 0.001, \\
& \sigma_{\mathrm{acc}}(K=1) / d_{p}=0.377 \pm 0.003, \\
& \sigma_{\mathrm{acc}}(K=-1) / d_{p}=0.405 \pm 0.001,
\end{aligned}
$$

i.e., adsorbate molecules of diameter $\sigma>\sigma_{\text {acc }}$ (the subindex "acc" refers to accessibility) will not be able to penetrate the porous media for a given type of disorder, in this particular case characterized by the parameter $K$.

In the analysis of the problem so far presented, we have considered spherical adsorbate molecules of diameter $\sigma$. This same diameter has been used to compute both the available volume and the accessibility to the porous media. It must be stressed that in some cases it could be convenient to perform the analysis of available volume and accessibility using different values for the adsorbate molecular size. When dealing with molecular adsorbates, one can find that the anisotropy and/or the flexibility of the molecules may facilitate the transit through bottlenecks within the pore structure, even when their effective (averaged) size might suggest the opposite.

\section{Computing the accessible volume: Voronoi/Delaunay tessellation}

In the preceding lines, we have learned how to estimate the limits of accessibility of adsorbate molecules to the porous material. Now, when analyzing the accessible volume to a given adsorbate molecule, we have to consider, as seen above, that certain regions in the porous media cannot be reached by adsorbates. In other words, we need to estimate the fraction of the available volume that can actually be occupied by the adsorbates entering the pores.

From the simulation point of view, we need a procedure to establish in an efficient way whether an available position is also accessible. The procedure must be easy and computationally inexpensive, since ultimately one would like to implement it on any grand canonical MC simulation of adsorption phenomena in disordered porous materials. In these systems one is likely to encounter isolated cavities that can- not be as easily spotted as in a crystalline porous material, such as a zeolite, or in more compact disordered materials with large porous structures, such as controlled pore glasses. $^{30}$

The strategy that we will follow is based on the Voronoi/ Delaunay tessellation. ${ }^{31}$ The system surface can be divided in small portions-Delaunay triangles (DTs) — using an appropriate procedure. We will show how, after this splitting of the surface, all the available positions inside a given portion of the surface (DT) - and consequently the surface (volume) corresponding to the DT in question-can be classified as either accessible or forbidden.

The distribution of the space (surface in our case) in domains associated with certain reference points is usually called tessellation. The Voronoi tessellation in our 2D system divides the surface in $N_{p}$ portions, with $N_{p}$ being the number of pillars. A given portion $k$ includes all the positions whose nearest pillar is the $k$ th one, and it is called Voronoi cell. The contour of the Voronoi cell is a convex polygon, each side of the polygon is located on the line that bisects the segment between pillar $k$ and one of its neighbors. We will define pillars $k$ and $l$ to be connected in the Voronoi construction if their corresponding Voronoi polygons (VPs) share a side. In disordered systems the vertices of the polygons correspond to the point where three VPs meet. (In ordered lattices four or more VPs can meet at certain vertices.)

The so-called Delaunay tessellation is related to (is dual to) the Voronoi tessellation. The polygon sides in the Delaunay case are defined by the lines connecting every pair of pillars whose VPs share a side. In disordered systems the Delaunay cells are triangles, and in our case a pillar can be found at each vertex of the DT.

Now, let us consider that we have found a certain position $\mathbf{r}_{i}$ in the porous system available for an adsorbate molecule of size $\sigma$. Let $\mathbf{r}_{i}$ be located inside a DT, $T_{k l m}$, where $k$, $l$, and $m$ designate the pillars (vertices) that define the triangle. First we want to analyze whether we can build a trajectory over available points to go from $\mathbf{r}_{i}$ to some other position right out of $T_{k l m}$. Such a trajectory will be impossible if the pillars $k, l$, and $m$ are placed in positions that imply the existence of the three barriers $L_{k l}, L_{k m}$, and $L_{l m}$ (i.e., if the length of the three triangle sides is less than $d_{p}$ $+\sigma)$. In this case the available positions inside $T_{k l m}$ will be isolated from other available positions outside the DT. On the contrary, if at least one of the sides of the DT is not a barrier, then it is possible to exit the DT from any available point in its interior.

According to the assumptions introduced above, we can readily establish whether a certain position is either accessible or blocked. In fact the connectivity properties of a given DT in the framework of a percolation analysis will determine whether the available positions inside the DT are accessible or not.

The connectivity analysis of the DTs is carried out as follows: once a Delaunay tessellation ${ }^{31}$ has been carried out on a given pillar configuration, we store for every pillar the information concerning the DTs that meet at the pillar (e.g., the coordinates of their vertices) and build up a neighbor list for each DT, bearing in mind that two DTs are considered 
TABLE IV. Limits of accessibility and fractions of available $\Phi_{\mathrm{av}}$ and accessible volume results for a model PILC using hard disk $(K=0)$ pillar disorder, a pillar density, $\rho_{p}=0.60 \mathrm{~nm}^{-2}$ (intercalated PTS). In the insertion of molecules a hard core diameter of $\sigma=0.32 \mathrm{~nm}$ was considered. The analysis of accessibility was performed by considering that two pillars at a distance less than $d_{p}+\sigma_{a}$ define a barrier with a value $\sigma_{a}=0.30 \mathrm{~nm}$.

\begin{tabular}{cccccccc}
\hline $\begin{array}{c}d_{p} \\
(\mathrm{~nm})\end{array}$ & $\rho_{p} d_{p}^{2}$ & $\sigma_{a} / d_{p}$ & $\bar{\sigma}_{\mathrm{per}}^{(1)} / d_{p}$ & $\bar{\sigma}_{\mathrm{per}}^{(2)} / d_{p}$ & $\bar{\sigma}_{\mathrm{per}} / d_{p}$ & $\Phi_{\mathrm{av}}$ & $\Phi_{\mathrm{acc}}$ \\
\hline 1.043 & 0.653 & 0.288 & 0.206 & 0.220 & 0.213 & 0.204 & 0.000 \\
1.000 & 0.600 & 0.300 & 0.258 & 0.275 & 0.267 & 0.245 & 0.011 \\
0.950 & 0.541 & 0.316 & 0.325 & 0.347 & 0.336 & 0.299 & 0.250 \\
0.900 & 0.486 & 0.333 & 0.406 & 0.433 & 0.420 & 0.346 & 0.338 \\
\hline \hline
\end{tabular}

neighbors whenever they share a side. Then we establish for each pair of neighboring DTs whether they are linked or not. A pair of neighboring DTs is considered to be linked if (and only if) their common side is not a barrier. Those links are then used to construct clusters of connected DTs and perform the usual cluster analysis. Obviously, the accessibility properties of the positions in a given DT will depend on the topology of its corresponding cluster. This means that the DTs belonging to nonpercolating clusters are considered to be not accessible. Also, if there is a cluster that percolates in the two directions of the space, all its DTs are considered to be accessible

In this way, we have split the area of our surface into a tessellation of triangles that can be classified as accessible or blocked. The computation of the accessible volume can be performed easily by a particle insertion procedure: for a given test position $\mathbf{r}_{\mathbf{i}}$, one determines the location of its nearest pillar $k$ (by which one additionally determines whether the position is available or not). Since we know which DTs meet at the pillar $k$, it is straightforward to assign $\mathbf{r}_{\mathbf{i}}$ to a certain DT, $T_{k l m}$. Now, an available position $\mathbf{r}_{i} \in T_{k l m}$ will also be accessible if (and only if) $T_{k l m}$ is an accessible DT.

\section{A PRACTICAL APPLICATION ON AN EXPERIMENTAL SYSTEM}

Guil and co-workers ${ }^{22}$ performed detailed calorimetric and volumetric adsorption studies on a series of PILCs resulting from $\mathrm{Al}$ intercalation on smectite clays from Benavila (BEN), PortoSanto (PTS), and Wyoming (WYO). The densities of pillars ${ }^{22}$ were estimated to be $0.60,0.68$, and 0.68 pillars $/ \mathrm{nm}^{2}$ for PTS, BEN, and WYO PILCs, respectively. Using simple geometrical considerations similar to those used in the analysis of the adsorption experiments on zeolites, ${ }^{12}$ and assuming a cylindrical geometry for the pillars, one arrives at a rough estimate of the pillar diameter $d_{p} \simeq 1.043 \mathrm{~nm}$. This calculation takes into account the microporous volume, as seen from $\mathrm{N}_{2}$ adsorption experiments, ${ }^{22}$ and the typical densities of the layers in these clays. ${ }^{32}$ The previous rough estimate of $d_{p}$ leads to reduced pillar densities, $\rho_{p} d_{p}^{2}$ of $0.65,0.74$, and 0.74 pillars $/ \mathrm{nm}^{2}$ for PTS, BEN, and WYO PILCs, respectively.

Using the PTS data, $\rho_{p} d_{p}^{2}=0.65$, we can compute the limit of accessibility for the hard disk disorder. For a system size $L / d_{p}=40$ we get $\bar{\sigma}_{\text {per }} \simeq 0.23 \mathrm{~nm}$ (see Table IV). Considering $\mathrm{N}_{2}$ as a standard adsorbate, its effective hard diameter is $\sigma_{\mathrm{N}_{2}} \simeq 0.36 \mathrm{~nm}$, by which $\sigma_{\mathrm{N}_{2}} / d_{p} \simeq 0.345$, and this means that for the hard disk disorder, not even $\mathrm{N}_{2}$ molecules could diffuse into the porous media, in clear contradiction with the experimental evidence. Moreover, in the cases of BEN and WYO PILCs, the value $d_{p}=1.043 \mathrm{~nm}$ will make the porous media inaccessible to $\mathrm{N}_{2}$ molecules even for an ordered triangular lattice (which exhibits the widest gates among the pillar structures studied above). Assuming the disordered structure of pillars for the PILCs, it could then be argued that pillars must cluster together so as to allow the entrance of the different probe adsorbates. In our theoretical modeling of the disorder, such a degree of grouping cannot be dealt with in a consistent way.

Therefore, in principle, our models of disorder disagree with the experimental findings. It is likely, however, that the aforementioned rough estimation of the pillar size is in part responsible for the failure of the model. The estimation of $d_{p}$ described above does not take into account the correlation between pillars, and it is implicitly based on a simple estimate of the fraction of accessible area, as given by

$$
\Phi_{\mathrm{acc}} \simeq 1-\frac{\pi \rho_{p} d_{p}^{2}}{4} .
$$

In our previous discussion we have seen that this equation only holds for low reduced density of pillars and low values of $\sigma / d_{p}$ (recall that $\sigma$ is the adsorbate size). The value of $\Phi_{\text {acc }}$ can be modified in two ways: when the pillars are grouped, the overlaps of their exclusion surfaces increase the available volume; at the same time the vicinity of a number of pillars can lead to a decrease in accessible volume due to the formation of inaccessible cavities.

Let us make some estimations of these effects. We have considered hard disk disorder $(K=0)$ and inserted disks (spheres) representing $\mathrm{N}_{2}$ molecules. We have taken as insertion diameter $\sigma_{i}=0.32 \mathrm{~nm}$ (slightly less than the LennardJones effective parameter for the $\mathrm{N}_{2}$ molecule). The accessibility diameter $\sigma_{\text {acc }}$ will be determined considering the Lennard-Jones parameter of two site models of $\mathrm{N}_{2}(0.331$ $\mathrm{nm}){ }^{33}$ taking then $\sigma_{a}=0.90 \sigma_{\mathrm{N}} \simeq 0.30 \mathrm{~nm}$. We have then performed calculations for different pillar diameters (with $\rho_{p}$ taken from Ref. 22) and computed the fractions of available and accessible areas. The calculations based on these assumptions are summarized in Table IV. In this table, one immediately observes that a $10 \%$ deviation in the estimation of the pillar diameters leads to substantial changes in the accessibility. This suggests that inaccuracies in these estimates might well be one of the sources of disagreement between our model results and the experiment, even without taking into account the possible effects of pillar aggregation. In this latter instance, if larger degrees of aggregation are needed one might resort to the use of a templating approach, such as that employed to model controlled pore glasses. ${ }^{30} \mathrm{In}$ this case, the pillar configuration can be generated using an equilibrium binary mixture modeled to exhibit a tendency to phase separate. If the thermodynamic conditions are close to the critical point (but not inside the two phase region) of the demixing transition, the sample will exhibit large inhomogeneities. Extracting configurations from a simulation in these thermodynamic states, and removing one of the components 
(the template), one would get a highly porous sample with a large degree of pillar aggregation. Actually, templating is also one of the possible paths for the synthesis of PILCs, ${ }^{3}$ and the process of calcination (and solvent removal) might also be ideally thought of as a form of templating. Alternatively, the interpillar interaction can be modified to incorporate a short range attraction and a long range repulsion. This type of interaction potential is known to be a good model for colloidal particles, which exhibit a large tendency to aggregate. In this regard, Imperio and Reatto ${ }^{34}$ showed how a simple model of this kind with competing interactions leads to impressive examples of pattern formation. Fluids with these types of interactions can constitute good precursors to obtain disordered highly porous materials such as PILCs.

In order to sum up the above discussion, the disagreement between our analysis and the conclusions drawn from the experiments can only be sorted out if the pillar diameters are known with sufficient accuracy and/or if direct information as to the pillar distribution is obtained from other experimental sources, such as neutron scattering. Work along these lines is currently under way.

Concerning future prospects, we intend to exploit the capabilities of ROZ approaches to model PILCs, a project which is well advanced in the pure $2 \mathrm{D}$ limit. ${ }^{35}$ Finally, a more robust comparison to the experimental results for the microporous volume would require the realization of grand canonical MC simulations considering explicitly the accessibility of the different pore cavities. Work in this line is also in progress.

In summary, we have presented a topological analysis of available and accessible volumes in simple models of PILCs. We have shown that the general considerations presented herein might be used to aid the interpretation of experimental results and single out those quantities which are essential for a useful modeling of these disordered systems.

\section{ACKNOWLEDGMENTS}

The authors gratefully acknowledge the support from the Dirección General de Investigación Científica y Técnica under Grant No. MAT2007-65711-C04-04 and from the Dirección General de Universidades e Investigación de la Comunidad de Madrid under Grant No. S0505/ESP/0299 and Program MOSSNOHO-CM.
${ }^{1}$ R. T. Yang and M. S. A. Baksh, AIChE J. 37, 679 (1991).

${ }^{2}$ A. Corma, Chem. Rev. (Washington, D.C.) 97, 2373 (1997).

${ }^{3}$ K. Ohtsuka, Chem. Mater. 9, 2039 (1997).

${ }^{4}$ S. Cheng, Catal. Today 49, 303 (1999).

${ }^{5}$ N. D. Hutson, M. J. Hoekstra, and R. T. Yang, Microporous Mesoporous Mater. 28, 447 (1999).

${ }^{6}$ A. Gil, M. A. Vicente, and L. M. Ganda, Microporous Mesoporous Mater. 34, 115 (2000).

${ }^{7}$ D. Plee, F. Borg, L. Gatineau, and J. J. Fripiat, J. Am. Chem. Soc. 107, 2362 (1985).

${ }^{8}$ T. J. Pinnavaia, S. D. Landau, M. S. Tzou, I. D. Johnson, and M. Lipsicas, J. Am. Chem. Soc. 107, 7222 (1985).

${ }^{9}$ A. Gil and G. Y. Cherkashinin, Adsorption 7, 265 (2001).

${ }^{10}$ J. P. Olivier and M. L. Occelli, J. Phys. Chem. B 105, 623 (2001).

${ }^{11}$ M. L. Occelli, J. P. Olivier, J. A. Perdigon-Melon, and A. Auroux, Langmuir 18, 9816 (2002).

${ }^{12}$ J. M. Guil, R. Guil-López, J. A. Perdigón-Melón, and A. Corma, Microporous Mesoporous Mater. 22, 269 (1998).

${ }^{13}$ A. De Stefanis, A. A. G. Tomlinson, Th. A. Steriotis, G. Ch. Charalambopoulou, and U. Keiderling, Appl. Surf. Sci. 253, 5633 (2007).

${ }^{14} \mathrm{~S}$. Torquato, Random Heterogeneous Materials, Microstructure and Macroscopic Properties (Springer, New York, 2002).

${ }^{15}$ W. G. Madden and E. D. Glandt, J. Stat. Phys. 51, 537 (1988).

${ }^{16}$ J. A. Given and G. Stell, J. Chem. Phys. 97, 4573 (1992).

${ }^{17}$ L. Zhang and P. R. van Tassel, J. Chem. Phys. 112, 3006 (2000).

${ }^{18}$ L. Sarkisov and P. R. Van Tassel, J. Chem. Phys. 123, 164706 (2005).

${ }^{19}$ O. Pizio and S. Sokolowski, Phys. Rev. E 56, R63 (1997).

${ }^{20}$ P. R. Van Tassel, Phys. Rev. E 60, R25 (1999).

${ }^{21}$ L. Sarkisov, J. Chem. Phys. 128, 044707 (2008).

${ }^{22}$ J. M. Guil, J. A. Perdigón-Melón, M. B. de Carvalho, A. P. Carvalho, and J. Pires, Microporous Mesoporous Mater. 51, 145 (2002).

${ }^{23}$ D. P. Landau and K. Binder, A Guide to Monte Carlo Simulations in Statistical Physics (Cambridge University, Cambridge, England, 2005).

${ }^{24}$ M. Allen and D. Tildesley, Computer Simulation of Liquids (Clarendon, Oxford, 1987).

${ }^{25}$ E. Lomba, C. Martín, N. G. Almarza, and F. Lado, Phys. Rev. E 71, 046132 (2005).

${ }^{26}$ G. Tarjus, P. Shaff, and J. Talbot, J. Stat. Phys. 63, 167 (1991).

${ }^{27}$ W. H. Press, S. A. Teukolsky, W. T. Vetterling, and B. P. Flannery, Numerical Recipes, 3rd ed. (Cambridge University, Cambridge, England, 2007).

${ }^{28}$ S. Sastry, D. S. Corti, P. G. Debenedetti, and F. H. Stillinger, Phys. Rev. E 56, 5524 (1997).

${ }^{29}$ D. Stauffer and A. Aharony, Introduction to Percolation Theory, 2nd ed. (Taylor \& Francis, London, 2003).

${ }^{30}$ L. D. Gelb and K. E. Gubbins, Langmuir 15, 305 (1999).

${ }^{31}$ M. Tanemura, T. Ogawa, and N. Ogita, J. Comput. Phys. 51, 191 (1983).

${ }^{32}$ G. D. Brunton, Clays Clay Miner. 36, 94 (1988).

${ }^{33}$ C. Martín, M. Lombardero, M. Alvarez, and E. Lomba, J. Chem. Phys. 102, 2092 (1995).

${ }^{34}$ A. Imperio and L. Reatto, J. Phys.: Condens. Matter 16, S3769 (2004).

${ }^{35}$ E. Lomba and J. J. Weis (unpublished). 